\title{
Flap Monitoring using Thermal Imaging Camera: A Contactless Method
}

\author{
Muhammad Jibran Rabbani, Asif Zubair Bhatti and Ahmed Shahzad \\ Department of Burn and Plastic Surgery, Shalamar Hospital, Lahore, Pakistan
}

\begin{abstract}
Objective: To determine the accuracy of the thermal imaging camera for the detection of vascular insult before the onset of permanent tissue damage.
\end{abstract}

Study Design: Descriptive study.

Place and Duration of Study: Department of Burn and Plastic Surgery, from July to December 2019.

Methodology: The thermal image was used as an adjunct to clinical assessment in 84 flaps. Both pedicled and free flaps were performed in the study with a frequency of $68(81 \%)$ and $16(19 \%)$, respectively. Flap monitoring was performed regularly for five days by same team of surgeons and nurses. Flap vascularity was assessed by temperature gradient colour coding of thermal image taking the clinical assessment like colour, temperature, capillary refill, as a gold standard. Sensitivity and specificity of the thermal camera were calculated.

Results: The age range of patients was from 5 years to 55 years. The diagnostic accuracy of thermal imaging camera was calculated to be $96.43 \%$ with a sensitivity of $98.7 \%$, specificity of $75 \%$. The positive predictive value was found to be $97.4 \%$, negative predictive value was $85.7 \%$, the false-negative value was $14.3 \%$, and the false-positive value was $2.6 \%$.

Conclusion: Thermal imaging camera is an effective and useful adjunct for clinical assessment, as it is an easy and contactless technique.

Key Words: Flir one, Flap monitoring, Thermal imaging camera.

How to cite this article: Rabbani MJ, Bhatti AZ, Shahzad A. Flap Monitoring using Thermal Imaging Camera: A Contactless Method. J Coll Physicians Surg Pak 2021; 31(06):703-706.

\section{INTRODUCTION}

Traditionally, flap monitoring in plastic surgery, either pedicled or free, is done subjectively by noting skin colour, skin turgor, temperature and capillary refill. Physical findings are subjected to errors, and this may lead to delay in picking vascular compromise and thus flap failure. ${ }^{1}$ Laboratory investigations like the $\mathrm{pH}$ of blood and flap glucose level monitoring have been introduced. ${ }^{2,3}$ Under discussion, medical devices are grouped as those which monitor vascularity at flap surface like Doppler flowmetry, near-infra-red, spectroscopy, acoustic Dopplerultrasound and visible light spectroscopy, and those that check vascular pedicle itself like Duplex ultrasonography, implantable Doppler. ${ }^{4,5}$ Recently, infrared thermography has been introduced in medical science for skin temperature monitoring, ${ }^{6}$ detection of inflammation, ${ }^{7}$ skin cancer $^{8}$ and breast cancer. $^{9}$

Correspondence to: Dr. Muhammad Jibran Rabbani, Department of Burn and Plastic Surgery, Shalamar Hospital, Lahore, Pakistan

E-mail: jibranrabbani@hotmail.com

Received: July 20, 2020; Revised: October 15, 2020;

Accepted: November 15, 2020

DOI: https://doi.org/10.29271/jcpsp.2021.06.703
These infrared thermography devices detect infrared radiation and convert them to images in which temperature values are proportional to pixel values. The obtained image can be taken as a marker for cutaneous blood flow. This technology has been applied to different areas of plastic surgery like burn depth assessmentand location of cutaneous perforators. ${ }^{10-12}$

Postoperatively, clinical methods of flap monitoring may be difficult in darker skin people. Thermal imaging camera can detect cutaneous perforators as well as an overall cutaneous blood supply. Limited research has been done to determine the accuracy of the thermal imaging camera in the identification of flap viability postoperatively.

The aim of this study was to use the utility of thermal imaging camera, as an adjunct to standard clinical methods used for postoperative flap monitoring.

\section{METHODOLOGY}

After taking the ethical approval and informed consent, all patients were enrolled, who underwent flap surgeries, either pedicled or free, in a duration of six months from July to December 2019. Patients with hypo or hyperthermia were excluded as this can produce artefacts taking normal body temperature range of 97 to $99^{\circ} \mathrm{F}^{13}{ }^{13} \mathrm{FLIR}$ ONE ${ }^{\circledR}$ (FLIR Systems, Inc., Wilsonville, Ore.) smartphone thermal imaging camera for 
detection of cutaneous blood flow was used. The area to be examined was kept exposed to room temperature for three minutes for acclimatisation. In case of a free flap, monitoring was carried out on hourly basis for first three days and then fourhourly basis for the next two days. While in the case of a pedicled flap, four-hourly monitoring was done for five days. Clinical assessment like flap colour, skin turgor, comparison of flap temperature with surround skin and capillary refill was carried out. In adjunct to this, a thermal image of the flap was obtained, and viability of the flap circulation was noted according totemperature gradient colour coding taking bright orange, yellow and white as well perfused while dark orange, red, blue or purple as poorly perfused or non-perfused areas. The same group of people, including the operating surgeon, did the assessment.

Diagnostic accuracy (TP + TN)/(TP + TN + FP + FN) was calculated using $2 \times 2$ table with clinical assessment as gold standard. True positive (TP - flap viable on thermal camera and clinical assessment as well), true negative (TN - flap non-viable on thermal camera and clinical assessment), false positive (FP - flap viable on thermal camera and not on clinical assessment) and false negative (FN - flap non-viable on thermal camera and but viable on clinical assessment) were noted. Accuracy was calculated in terms of sensitivity (ability of thermal imaging in detection of flap viability, TP / (TP + FN) x 100), specificity (ability of thermal imaging in detection of flap non-viability. TN / (TN + FP) $x$ 100), positive predictive value (PPV - Proportion of flap viability in all positive cases. TP / (TP + FP) x 100) and negative predictive value (NPV - Proportion of flap non-viability, among all negative cases, and TN / (TN + FN) x 100). Mean, and the standard deviation was calculated for numerical variables like age. Frequencies and percentages were calculated for categorical variables like gender and flap type (pedicled/free). Chi-square test was applied with $p$-value $\leq 0.05$ considered as significant. The collected data was entered and analysed using SPSS version 20 .

\section{RESULTS}

A total of 84 patients were included. The age range was from 5 years to 55 years, with a mean age of $29.21 \pm 11.744$ years, males $(70,83.3 \%)$ outnumbering females $(14,16.7 \%)$. Both pedicled and free flaps were performed in the study with a frequency of 68 $(81 \%)$ and $16(19 \%)$, respectively. Partial or complete necrosis was seen in $8(9.5 \%)$ flaps. Poor or no perfusion were identified in all necrotic flaps by clinical assessment. However, the thermal imaging camera showed non-viability in 7 (8.3\%) cases. The thermal camera was unable to detect one case of poor perfusion until the flap was completely dead (Figure 2). The diagnostic accuracy of the thermal imaging camera was calculated to be $96.43 \%$ with a sensitivity of $98.7 \%$, specificity of $75 \%$. The positive predictive value was found to be $97.4 \%$, negative predictive value was $85.7 \%$, the false-negative value was $14.3 \%$, and the false-positive value was $2.6 \%$ (Table I).

\section{DISCUSSION}

The occurrence of flap failure depends on multiple factors irrespective of the postoperative monitoring method used. Flap failure is mostcommon in the first three days of surgery. ${ }^{14}$ Further- more, the likelihood of flap salvage is improved if early intervention is taken after the onset of the features of vascular compromise. ${ }^{15}$ The most crucial step is to detect the vascular compromise before the onset of permanent tissue damage. About 68 $75 \%$ flaps can be salvaged if the re-vascularisation is achieved within an hour of vascular insult. ${ }^{16}$

Detection of vascular insult predominantly depends on the method of monitoring. The methods used should be easy, accurate, free of hassle, reliable and quick. There are more chances of error in clinical assessment as it is subjective that relies on the observer's ability to pick the findings. Furthermore, there is a delay in the appearance of cutaneous features and the actual onset of vascular blockage. Many technologies are used to serve this purpose. Implantable Doppler, Duplex, Doppler flowmetry, near-infrared, and spectroscopy are different instruments that have been introduced. ${ }^{4}$ All these instruments have not gained much popularity due to either inaccessibility or their cost. ${ }^{5}$

Thermal imaging camera has recently been introduced in medical science. This technology converts infrared electromagnetic radiations to a pictorial format that are proportional to the temperature where different colours are specific for a temperature gradient. Overall, brighter images are produced with a default colour range from dark blue that represents the coolest body part to garish yellow or white that represent the hottest part. Different shades of red and orange show body parts with intermediate temperature levels. A thermographic camera (aka infrared camera or thermal imaging camera) uses infrared radiation to form an image. The operating wavelength of the thermal camera is $14,00 \mathrm{~nm},{ }^{17}$ which differs it from an ordinary light digital camera (wavelength of $400-700 \mathrm{~nm}$ ). ${ }^{18} \mathrm{FLIR}$ ONE ${ }^{\circledR}$ is a low cost imaging device that couples with micro-USB port of Android or lightening port of iOS smartphones. It uses a second light camera to capture still or moving real time image forcomparison. Furthermore, absolute temperature estimation is also possible for a specific spot.

It was aimed to identify the accuracy of the thermal imaging camera in terms of postoperative flap viability and used as an adjunct to the traditional method of clinical assessment. The flap, either pedicled or free, was monitored for five days postoperatively. It was found the sensitivity of this instrument to identify vascularity of flap to be $98.7 \%$ and specificity of $75 \%$. Vascular compromise was not detected by the thermal camera but was picked earlier clinically at the second postoperative day in one case. The cause of partial flap loss, in thiscase, was venous insufficiency. This deficiency in detection may be attributed to the fact that there is a delay in temperature changes in the case of venous obstruction.

Maren et al. conducted a study on monitoring of free flaps for oropharyngeal reconstruction by thermal imaging in 2015 . They calculated the temperature differences and examined the thermal images in pre-operative, intra-operative (both pre-anastomosis and post-anastomosis) and postoperative course and found significant temperature difference before and after vascular anastomosis of the flap. 
Table I: Thermal image assessment vs. clinical assessment cross tabulation.

\begin{tabular}{|c|c|c|c|c|c|}
\hline & & & \multicolumn{2}{|c|}{ Clinical assessment } & \multirow{2}{*}{ Total } \\
\hline & & & Viable & Non-viable & \\
\hline \multirow{6}{*}{$\begin{array}{l}\text { Thermal image } \\
\text { assessment }\end{array}$} & \multirow{3}{*}{ Viable } & Count & 75 & 2 & 77 \\
\hline & & $\%$ within Thermal Image Assessment & $97.4 \%$ & $2.6 \%$ & $100.0 \%$ \\
\hline & & $\%$ within clinical assessment & $98.7 \%$ & $25.0 \%$ & $91.7 \%$ \\
\hline & \multirow{3}{*}{ Non-viable } & Count & 1 & 6 & 7 \\
\hline & & $\%$ within thermal image assessment & $14.3 \%$ & $85.7 \%$ & $100.0 \%$ \\
\hline & & $\%$ within clinical assessment & $1.3 \%$ & $75.0 \%$ & $8.3 \%$ \\
\hline \multirow{3}{*}{\multicolumn{2}{|c|}{ Total }} & Count & 76 & 8 & 84 \\
\hline & & $\%$ within thermal image assessment & $90.5 \%$ & $9.5 \%$ & $100.0 \%$ \\
\hline & & $\%$ within clinical assessment & $100.0 \%$ & $100.0 \%$ & $100.0 \%$ \\
\hline \multicolumn{2}{|l|}{$\begin{array}{l}\text { Chi-square test } \\
\text { Likelihood ratio }\end{array}$} & \multicolumn{4}{|l|}{$\begin{array}{l}<0.001 \\
<0.001\end{array}$} \\
\hline
\end{tabular}

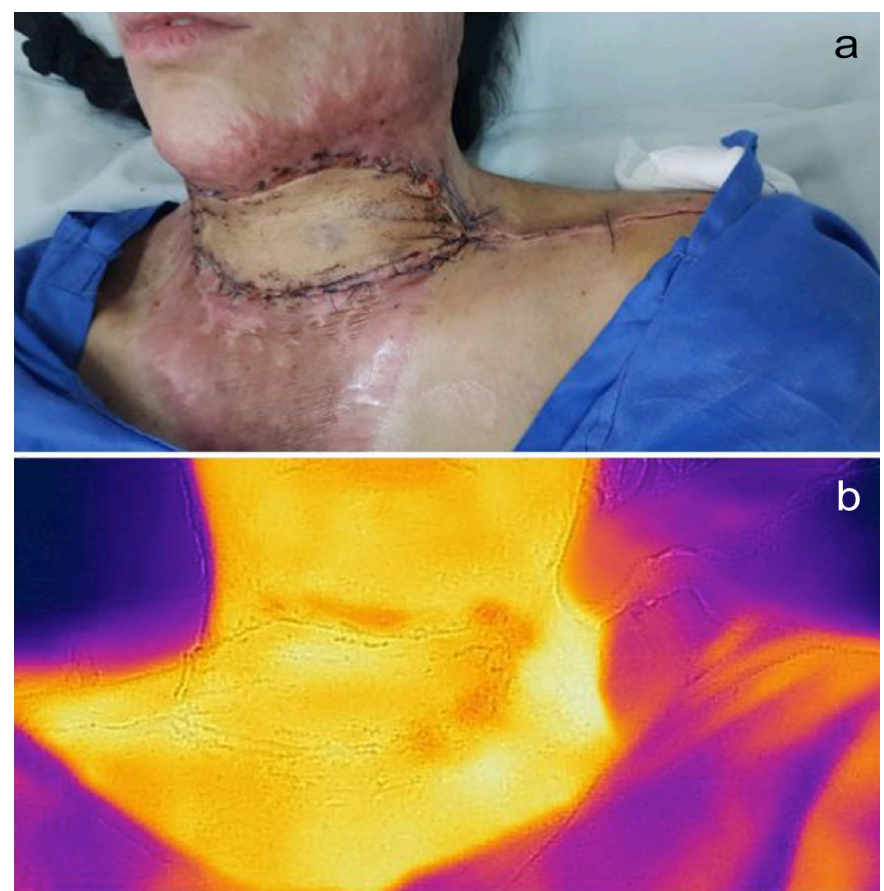

Figure 1: First postoperative day after post-burn neck contracture release and resurfaced with supraclavicular artery flap: (a) Clinically viable flap; (b) Flap viability is seen on thermal image.

They found it a useful tool for the identification of vascular compromise. They also suggested that failure of flap perfusion is likely, if there is no substantial decrease in the difference of flap and surrounding temperature immediately after the vascular anastomosis. ${ }^{19}$ They used endoscopic infrared thermographic camera and relied more on temperature difference than the thermal image itself.

Xavier et al. used a thermographic camera to map colour and temperature differences after ligation of the inferior superficial epigastric vessel of one side using the contralateral artery as a control in 20 rats. They found immediate disappearance of hot spot corresponding to the artery after ligation before skin showed any change of vascular compromise. Similarly, thermographic changes appeared after venous ligation before the macroscopic appearance of congestion. They concluded that thermographic camera can detect vascular insufficiency before the appearance of clinical features. ${ }^{20}$

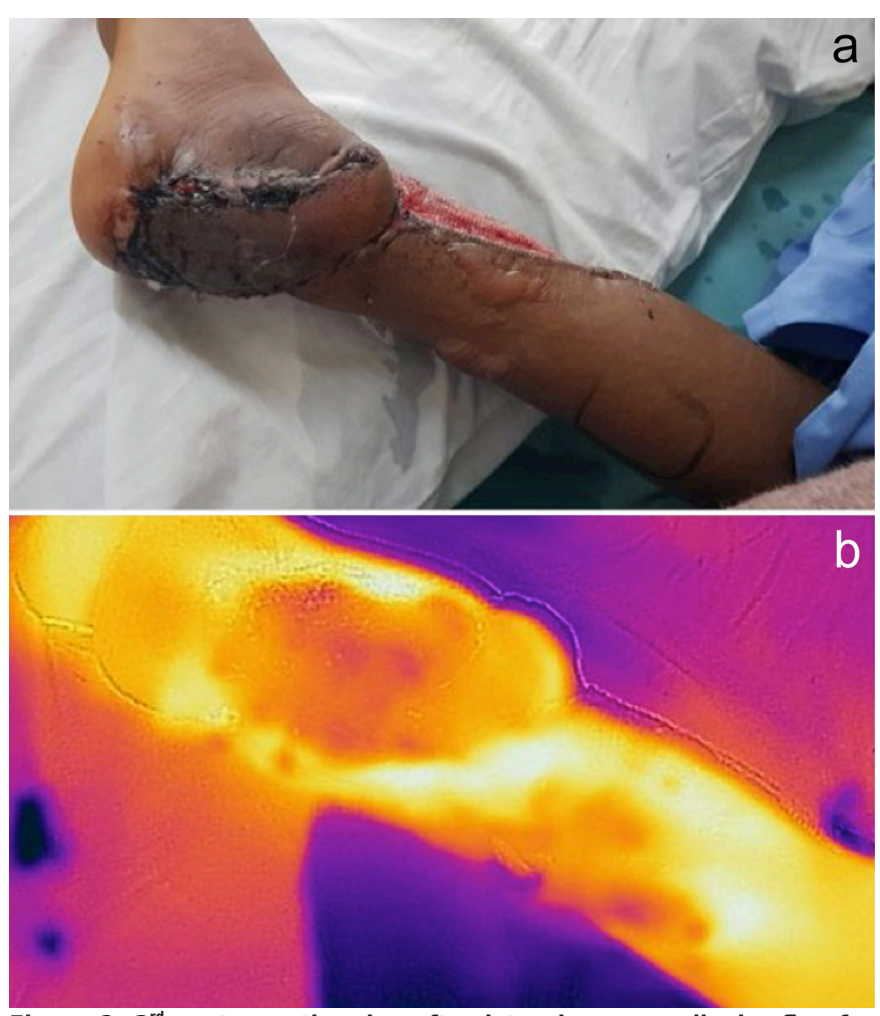

Figure 2: $3^{\text {rd }}$ postoperative day after lateral supra-malleolar flap for coverage of heel defect. (a) Distal half of flap necrosis on clinical assessment; (b) Flap necrosis can be identified on thermal image as darker colours showing poor perfusion.

Many factors can affect the results of a thermal image obtained, such as local inflammation, body temperature and respiratory activity. External factors like room temperature and air humidity can also have significant influence. It is recommended to expose the body part to be examined to the room environment for acclimatisation.

This study showed that thermal imaging camera can be used in adjunct to clinical assessment to identify vascular compromise of the flap at earliest. However, the authors' did not focus on the flap salvage interventions, in case, any perfusion issue is identified. The authors will comment on the time interval between vascular insufficiency identified on thermal camera and appearance of clinical features in their future study. 


\section{CONCLUSION}

Thermal imaging camera is a hand-held useful adjunct imaging technique for postoperative flap monitoring for it is being easy, quick, non-invasive and reliable. Above all, it is a contactless method that can prevent cross-infection. Subjective errors by observers can be eliminated.

\section{ETHICAL APPROVAL:}

Ethical approval was taken from the Institutional Review Board of Shalamar Medical and Dental College, Lahore before the initiation of the research work.

\section{PATIENTS' CONSENT:}

Informed consents were taken from all the patients to publish the results obtained from the study.

\section{CONFLICT OF INTEREST}

The authors declared no conflict of interest, especially regarding the use of a medical device.

\section{AUTHORS' CONTRIBUTION:}

MJR: Acquired data, data analysis, results preparation, writeup, review, manuscript revision.

AZB, AS: Acquired data, results analysis, results preparation, manuscript review.

\section{REFERENCES}

1. de Weerd L, Mercer JB, Setså LB. Intraoperative dynamic infrared thermography and free-flap surgery. Ann Plas Surg 2006; 57(3):279-84. doi: 10.1097/01.sap.0000218579. 17185.c9.

2. Chen K-T, Mardini S, Chuang DCC, Lin CH, Cheng MH, Lin YT, et al. Timing of presentation of the first signs of vascular compromise dictates the salvage outcome of free flap transfers. Plas Reconstr Surg 2007; 120(1):187-95. doi: 10.1097/01.prs.0000264077.07779.50.

3. Salgado CJ, Moran SL, Mardini S. Flap monitoring and patient management. Plast Reconstr Surg 2009; 124(6S): e295-e302. doi: 10.1097/PRS.0b013e3181bcf07b.

4. Chao AH, Lamp S. Current approaches to free flap monitoring. Plas Surg Nurs 2014; 34(2):52-6. doi: 10.1097/ PSN.0000000000000037.

5. Smit JM, Zeebregts CJ, Acosta R, Werker PM. Advancements in free flap monitoring in the last decade: A critical review. Plas Reconstr Surg 2010; 125(1):177-85. doi: 10.1097/ PRS.0b013e3181c49580.

6. Chang K, Yoon S, Sheth N, Seidel M, Antalek M, Ahad J, et al. Rapid vs. delayed infrared responses after ischemia reveal recruitment of different vascular beds. Quant infrared Thermogr J 2015; 12(2):173-83. doi: 10.1080/ 17686733. 2015.1046677.

7. Jin C, Yang Y, Xue ZJ, Liu KM, Liu J. Automated analysis method for screening knee osteoarthritis using medical infrared thermography. J Med Biological Engineering 2013; 33(5):471-7.

8. Çetingül MP, Herman C. Quantification of the thermal signature of a melanoma lesion. Int J Therm Sci 2011; 50(4):421-31.

9. Nicandro CR, Efrén MM, María Yaneli AA, Enrique MDCM, Héctor Gabriel AM, Nancy PC, et al. Evaluation of the diagnostic power of thermography in breast cancer using bayesian network classifiers. Comput Math Methods Med 2013; 2013 doi: 10.1155/2013/264246.

10. Hardwicke J, Thomson R, Bamford A, Moiemen N. A pilot evaluation study of high resolution digital thermal imaging in the assessment of burn depth. Burns 2013; 39(1):76-81. doi: 10.1016/j.burns.2012.03.014.

11. Renkielska A, Kaczmarek M, Nowakowski A, Grudziński J, Czapiewski P, Krajewski A, et al. Active dynamic infrared thermal imaging in burn depth evaluation. J Burn Care Res 2014; 35(5):e294-e303. doi: 10.1097/BCR.0000000000 000059.

12. Sheena $Y$, Jennison T, Hardwicke JT, Titley OG. Detection of perforators using thermal imaging. Plas Reconstr Surg 2013; 132(6):1603-10.

13. Vorvick L, Zieve D. ADAM editorial team (2018) Body temperature norms: MedlinePlus Medical Encyclopedia. Medlineplus. gov.

14. Chao AH, Meyerson J, Povoski SP, Kocak E. A review of devices used in the monitoring of microvascular free tissue transfers. Expert Rev Med Devices 2013; 10(5):649-60. doi: 10.1586/17434440.2013.827527.

15. Kroll SS, Schusterman MA, Reece GP, Miller MJ, Evans GR, Robb GL, et al. Timing of pedicle thrombosis and flap loss after free-tissue transfer. Plastic Reconstr Surg 1996; 98(7):1230-3. doi: 10.1097/00006534-199612000-00017.

16. Brown J, Devine J, Magennis $P$, Sillifant $P$, Rogers $S$, Vaughan E. Factors that influence the outcome of salvage in free tissue transfer. BrJ Oral Maxillofac Surg 2003; 41(1):16-20. doi: 10.1016/s0266-4356(02)00260-7.

17. Battalwar P, Gokhale J, Bansod U. Infrared thermography and IR camera. History 2015; 1(2).

18. Bokolonga E, Hauhana M, Rollings N, Aitchison D, Assaf MH, Das SR, et al. A compact multispectral image capture unit for deployment on drones. Int Instr Measurement Technol Confer Proceeds; 2016: IEEE.

19. Just M, Chalopin C, Unger M, Halama D, Neumuth T, Dietz A, et al. Monitoring of microvascular free flaps following oropharyngeal reconstruction using infrared thermography: first clinical experiences. Eur Arch Otorhinolaryngolo 2016; 273(9):2659-67. doi: 10.1007/s00405-015-3780-9.

20. Tenorio X, Mahajan AL, Wettstein R, Harder Y, Pawlovski M, Pittet $B$. Early detection of flap failure using a new thermographic device. J Surg Res 2009; 151(1):15-21. doi: 10.1016/j.jss.2008.03.001. 